Article

\title{
On a Reverse Half-Discrete Hardy-Hilbert's Inequality with Parameters
}

\author{
Bicheng Yang ${ }^{1}$, Shanhe $\mathrm{Wu}^{2, *}$ (D) and Aizhen Wang ${ }^{3}$
}

1 Institute of Applied Mathematics, Longyan University, Longyan 364012, China; bcyang@gdei.edu.cn

2 Department of Mathematics, Longyan University, Longyan 364012, China

3 Department of Mathematics, Guangdong University of Education, Guangzhou 510303, China;

ershimath@163.com

* Correspondence: shanhewu@163.com

Received: 22 August 2019; Accepted: 29 October 2019; Published: 4 November 2019

Abstract: By means of the weight functions, the idea of introduced parameters, and the Euler-Maclaurin summation formula, a reverse half-discrete Hardy-Hilbert's inequality and the reverse equivalent forms are given. The equivalent statements of the best possible constant factor involving several parameters are considered. As applications, two results related to the case of the non-homogeneous kernel and some particular cases are obtained.

Keywords: weight function; half-discrete Hardy-Hilbert's inequality; parameter; Euler-Maclaurin summation formula; reverse inequality

MSC: 26D15; 26D10; 26A42

\section{Introduction}

If $0<\sum_{m=1}^{\infty} a_{m}^{2}<\infty$ and $0<\sum_{n=1}^{\infty} b_{n}^{2}<\infty$, then we have the following discrete Hilbert's inequality with the best possible constant factor $\pi$ (cf., [1], Theorem 315):

$$
\sum_{m=1}^{\infty} \sum_{n=1}^{\infty} \frac{a_{m} b_{n}}{m+n}<\pi\left(\sum_{m=1}^{\infty} a_{m}^{2} \sum_{n=1}^{\infty} b_{n}^{2}\right)^{1 / 2}
$$

Correspondingly, if $0<\int_{0}^{\infty} f^{2}(x) d x<\infty$ and $0<\int_{0}^{\infty} g^{2}(y) d y<\infty$, we still have the following Hilbert's integral inequality (cf., [1], Theorem 316):

$$
\int_{0}^{\infty} \int_{0}^{\infty} \frac{f(x) g(y)}{x+y} d x d y<\pi\left(\int_{0}^{\infty} f^{2}(x) d x \int_{0}^{\infty} g^{2}(y) d y\right)^{1 / 2}
$$

where the constant factor $\pi$ is the best possible.

As is known to us, Inequalities (1) and (2) and their extensions with conjugate exponents as well as independent parameters play an important role in analysis and their applications (cf., [2-13]).

Concerning with Inequalities (1) and (2), we have the following half-discrete Hilbert-type inequality (cf., [1], Theorem 351):

If $K(x)(x>0)$ is a decreasing function and $p>1, \frac{1}{p}+\frac{1}{q}=1,0<\phi(s)=\int_{0}^{\infty} K(x) x^{s-1} d x<\infty$, $f(x) \geq 0,0<\int_{0}^{\infty} f^{p}(x) d x<\infty$, then

$$
\sum_{n=1}^{\infty} n^{p-2}\left(\int_{0}^{\infty} K(n x) f(x) d x\right)^{p}<\phi^{p}\left(\frac{1}{q}\right) \int_{0}^{\infty} f^{p}(x) d x .
$$


In recent years, some new extensions of the Inequality (3) were provided in [14-19].

In 2006, with the help of the Euler-Maclaurin summation formula, Krnic et al. [20] gave an extension of (1) with the kernel $\frac{1}{(m+n)^{\lambda}}(0<\lambda \leq 14)$. In 2019, Adiyasuren et al. [21] considered an extension of (1) with $p, q>1\left(\frac{1}{p}+\frac{1}{q}=1\right)$ involving the partial sums. In 2016-2017, by using the weight functions, Hong [22,23] considered some equivalent statements of the extensions of (1) and (2) with several parameters. Some related works can be found in [24-26].

In this paper, following the way of $[20,22]$, by using the weight functions, the idea of introduced parameters, and the Euler-Maclaurin summation formula, a reverse half-discrete Hardy-Hilbert's inequality with the homogeneous kernel $\frac{1}{(x+n)^{\lambda}}(0<\lambda \leq 5)$ and the reverse equivalent forms are established. The equivalent statements of the best possible constant factor related to several parameters are presented. As applications, two corollaries related to the case of the non-homogeneous kernel and some particular cases are obtained.

\section{Some Lemmas}

In what follows, we assume that

$$
0<p<1(q<0), \frac{1}{p}+\frac{1}{q}=1, \lambda \in(0,5], \sigma \in(0,2] \cap(0, \lambda), \mu \in(0, \lambda),
$$

$f(x) \geq 0\left(x \in R_{+}=(0, \infty)\right), a_{n} \geq 0(n \in \mathrm{N}=\{1,2, \cdots\})$ satisfying

$$
0<\int_{0}^{\infty} x^{p\left[1-\left(\frac{\lambda-\sigma}{p}+\frac{\mu}{q}\right)\right]-1} f^{p}(x) d x<\infty \text { and } 0<\sum_{n=1}^{\infty} n^{q\left[1-\left(\frac{\sigma}{p}+\frac{\lambda-\mu}{q}\right)\right]-1} a_{n}^{q}<\infty .
$$

Lemma 1. Define a weight function by

$$
\omega(\sigma, x):=x^{\lambda-\sigma} \sum_{n=1}^{\infty} \frac{n^{\sigma-1}}{(x+n)^{\lambda}}\left(x \in \mathrm{R}_{+}\right) .
$$

Then, we have

$$
B(\sigma, \lambda-\sigma)\left(1-\rho_{\sigma}(x)\right)<\omega(\sigma, x)<B(\sigma, \lambda-\sigma)\left(x \in \mathrm{R}_{+}\right),
$$

where, $\rho_{\sigma}(x):=\frac{\left(1+\theta_{x}\right)^{-\lambda}}{\sigma B(\sigma, \lambda-\sigma)} \frac{1}{x^{\sigma}}=O\left(\frac{1}{x^{\sigma}}\right) \in(0,1)\left(\theta_{x} \in\left(0, \frac{1}{x}\right) ; x>0\right) . B(u, v):=\int_{0}^{\infty} \frac{t^{u-1}}{(1+t)^{u+v}} d t(u, v>0)$ is the beta function.

Proof. For fixed $x>0$, we set function $g_{x}(t):=\frac{t^{\sigma-1}}{(x+t)^{\lambda}}(t>0)$. Using the Euler-Maclaurin summation formula (cf., [20]), for $\rho(t):=t-[t]-\frac{1}{2}$, we have

$$
\begin{aligned}
& \sum_{n=1}^{\infty} g_{x}(n)=\int_{1}^{\infty} g_{x}(t) d t+\frac{1}{2} g_{x}(1)+\int_{1}^{\infty} \rho(t) g_{x}^{\prime}(t) d t=\int_{0}^{\infty} g_{x}(t) d t-h(x) \\
& h(x):=\int_{0}^{1} g_{x}(t) d t-\frac{1}{2} g_{x}(1)-\int_{1}^{\infty} \rho(t) g_{x}^{\prime}(t) d t .
\end{aligned}
$$

Thus, we obtain $-\frac{1}{2} g_{x}(1)=\frac{-1}{2(x+1)^{\lambda}}$,

$$
\begin{aligned}
\int_{0}^{1} g_{x}(t) d t & =\int_{0}^{1} \frac{t^{\sigma-1}}{(x+t)^{\lambda}} d t=\frac{1}{\sigma} \int_{0}^{1} \frac{d t^{\sigma}}{(x+t)^{\lambda}}=\left.\frac{1}{\sigma} \frac{t^{\sigma}}{(x+t)^{\lambda}}\right|_{0} ^{1}+\frac{\lambda}{\sigma} \int_{0}^{1} \frac{t^{\sigma} d t}{(x+t)^{\lambda+1}} \\
& =\frac{1}{\sigma} \frac{1}{(x+1)^{\lambda}}+\frac{\lambda}{\sigma(\sigma+1)} \int_{0}^{1} \frac{d t^{\sigma+1}}{(x+t)^{\lambda+1}}
\end{aligned}
$$




$$
\begin{gathered}
>\frac{1}{\sigma} \frac{1}{(x+1)^{\lambda}}+\frac{\lambda}{\sigma(\sigma+1)}\left[\frac{t^{\sigma+1}}{(x+t)^{\lambda+1}}\right]_{0}^{1}+\frac{\lambda(\lambda+1)}{\sigma(\sigma+1)(x+1)^{\lambda+2}} \int_{0}^{1} t^{\sigma+1} d t \\
=\frac{1}{\sigma} \frac{1}{(x+1)^{\lambda}}+\frac{\lambda}{\sigma(\sigma+1)} \frac{1}{(x+1)^{\lambda+1}}+\frac{\lambda(\lambda+1)}{\sigma(\sigma+1)(\sigma+2)} \frac{1}{(x+1)^{\lambda+2}}, \\
-g_{x}^{\prime}(t)=-\frac{(\sigma-1) t^{\sigma-2}}{(x+t)^{\lambda}}+\frac{\lambda t^{\sigma-1}}{(x+t)^{\lambda+1}}=\frac{(1-\sigma) t^{\sigma-2}}{(x+t)^{\lambda}}+\frac{\lambda t^{\sigma-2}}{(x+t)^{\lambda}}-\frac{\lambda x t^{\sigma-2}}{(x+t)^{\lambda+1}} \\
=\frac{(\lambda+1-\sigma) t^{\sigma-2}}{(x+t)^{\lambda}}-\frac{\lambda x t^{\sigma-2}}{(x+t)^{\lambda+1}} .
\end{gathered}
$$

For $0<\sigma \leq 2, \sigma<\lambda \leq 5$, we find

$$
(-1)^{i} \frac{d^{i}}{d t^{i}}\left[\frac{t^{\sigma-2}}{(x+t)^{\lambda}}\right]>0,(-1)^{i} \frac{d^{i}}{d t^{i}}\left[\frac{t^{\sigma-2}}{(x+t)^{\lambda+1}}\right]>0(t>0 ; i=0,1,2,3),
$$

and then by using the Euler-Maclaurin summation formula (cf., [20]), we find

$$
\begin{gathered}
(\lambda+1-\sigma) \int_{1}^{\infty} \rho(t) \frac{t^{\sigma-2}}{(x+t)^{\lambda}} d t>-\frac{\lambda+1-\sigma}{12(x+1)^{\lambda}}, \\
-x \lambda \int_{1}^{\infty} \rho(t) \frac{t^{\sigma-2}}{(x+t)^{\lambda+1}} d t>\frac{x \lambda}{12(x+1)^{\lambda+1}}-\frac{x \lambda}{720}\left[\frac{t^{\sigma-2}}{(x+t)^{\lambda+1}}\right]^{\prime \prime} \\
>\frac{(x+1) \lambda-\lambda}{12(x+1)^{\lambda+1}}-\frac{(x+1) \lambda}{720}\left[\frac{(\lambda+1)(\lambda+2)}{(x+1)^{\lambda+3}}+\frac{2(\lambda+1)(2-\sigma)}{(x+1)^{\lambda+2}}+\frac{(2-\sigma)(3-\sigma)}{(x+1)^{\lambda+1}}\right] \\
=\frac{\lambda}{12(x+1)^{\lambda}}-\frac{\lambda}{12(x+1)^{\lambda+1}}-\frac{\lambda}{720}\left[\frac{(\lambda+1)(\lambda+2)}{(x+1)^{\lambda+2}}+\frac{2(\lambda+1)(2-\sigma)}{(x+1)^{\lambda+1}}+\frac{(2-\sigma)(3-\sigma)}{(x+1)^{\lambda}}\right] .
\end{gathered}
$$

Hence, we have

$$
h(x)>\frac{h_{1}}{(x+1)^{\lambda}}+\frac{\lambda h_{2}}{(x+1)^{\lambda+1}}+\frac{\lambda(\lambda+1) h_{3}}{(x+1)^{\lambda+2}}
$$

where, $h_{1}:=\frac{1}{\sigma}-\frac{1}{2}-\frac{1-\sigma}{12}-\frac{\lambda(2-\sigma)(3-\sigma)}{720}, h_{2}:=\frac{1}{\sigma(\sigma+1)}-\frac{1}{12}-\frac{(\lambda+1)(2-\sigma)}{720}$, and

$$
h_{3}:=\frac{1}{\sigma(\sigma+1)(\sigma+2)}-\frac{\lambda+2}{720}
$$

For $\lambda \in(0,5], \frac{\lambda}{720}<\frac{1}{24}, \sigma \in(0,2]$, it follows that

$$
h_{1}>\frac{1}{\sigma}-\frac{1}{2}-\frac{1-\sigma}{12}-\frac{(2-\sigma)(3-\sigma)}{24}=\frac{24-20 \sigma+7 \sigma^{2}-\sigma^{3}}{24 \sigma}>0 .
$$

In fact, setting $g(\sigma):=24-20 \sigma+7 \sigma^{2}-\sigma^{3}(\sigma \in(0,2])$, we obtain

$$
g^{\prime}(\sigma)=-20+14 \sigma^{2}-3 \sigma^{2}=-3\left(\sigma-\frac{7}{3}\right)^{2}-\frac{11}{3}<0
$$

and then we obtain $h_{1}>\frac{g(\sigma)}{24 \sigma} \geq \frac{g(2)}{24 \sigma}=\frac{4}{24 \sigma}>0(\sigma \in(0,2])$.

We observe that $h_{2}>\frac{1}{6}-\frac{1}{12}-\frac{12}{360}=\frac{1}{20}>0$, and $h_{3} \geq \frac{1}{24}-\frac{7}{720}=\frac{23}{720}>0$. Hence, we deduce that $h(x)>0$, and thus we have

$$
\begin{aligned}
\omega(\sigma, x) & =x^{\lambda-\sigma} \sum_{n=1}^{\infty} g_{x}(n)<x^{\lambda-\sigma} \int_{0}^{\infty} g_{x}(t) d t \\
& =x^{\lambda-\sigma} \int_{0}^{\infty} \frac{t^{\sigma-1} d t}{(x+t)^{\lambda}}=\int_{0}^{\infty} \frac{u^{\sigma-1} d u}{(1+u)^{\lambda}}=B(\sigma, \lambda-\sigma)
\end{aligned}
$$


On the other-hand, we also have

$$
\begin{aligned}
\sum_{n=1}^{\infty} g_{x}(n) & =\int_{1}^{\infty} g_{x}(t) d t+\frac{1}{2} g_{x}(1)+\int_{1}^{\infty} \rho(t) g_{x}^{\prime}(t) d t \\
& =\int_{1}^{\infty} g_{x}(t) d t+H(x) \\
H(x) & :=\frac{1}{2} g_{x}(1)+\int_{1}^{\infty} \rho(t) g_{x}^{\prime}(t) d t .
\end{aligned}
$$

We obtain $\frac{1}{2} g_{x}(1)=\frac{1}{2(x+1)^{\lambda}}$ and

$$
g_{x}^{\prime}(t)=\frac{-(\lambda+1-\sigma) t^{\sigma-2}}{(x+t)^{\lambda}}+\frac{\lambda x t^{\sigma-2}}{(x+t)^{\lambda+1}} .
$$

For $\sigma \in(0,2] \cap(0, \lambda), 0<\lambda \leq 5$, by the Euler-Maclaurin summation formula, we obtain

$$
\begin{gathered}
-(\lambda+1-\sigma) \int_{1}^{\infty} \rho(t) \frac{t^{\sigma-2}}{(x+t)^{\lambda}} d t>0, \\
x \lambda \int_{1}^{\infty} \rho(t) \frac{t^{\sigma-2}}{(x+t)^{\lambda+1}} d t \\
>-\frac{x \lambda}{12(x+1)^{\lambda+1}}=-\frac{(x+1) \lambda-\lambda}{12(x+1)^{\lambda+1}}=\frac{-\lambda}{12(x+1)^{\lambda}}+\frac{\lambda}{12(x+1)^{\lambda+1}}>\frac{-\lambda}{12(x+1)^{\lambda}} .
\end{gathered}
$$

Hence, we have

$$
H(x)>\frac{1}{2(x+1)^{\lambda}}-\frac{\lambda}{12(x+1)^{\lambda}}=\frac{6-\lambda}{12(x+1)^{\lambda}}>0
$$

and then

$$
\begin{aligned}
\omega(\sigma, x) & =x^{\lambda-\sigma} \sum_{n=1}^{\infty} g_{x}(n)>x^{\lambda-\sigma} \int_{1}^{\infty} g_{x}(t) d t \\
& =x^{\lambda-\sigma} \int_{0}^{\infty} g_{x}(t) d t-x^{\lambda-\sigma} \int_{0}^{1} g_{x}(t) d t \\
& =B(\sigma, \lambda-\sigma)\left[1-\frac{1}{B(\sigma, \lambda-\sigma)} \int_{0}^{\frac{1}{x}} \frac{u^{\sigma-1}}{(1+u)^{\lambda}} d u\right]>0 .
\end{aligned}
$$

By the integral mid-value theorem, we find

$$
\int_{0}^{\frac{1}{x}} \frac{u^{\sigma-1}}{(1+u)^{\lambda}} d u=\frac{1}{\left(1+\theta_{x}\right)^{\lambda}} \int_{0}^{\frac{1}{x}} u^{\sigma-1} d u=\frac{1}{\sigma\left(1+\theta_{x}\right)^{\lambda}} \frac{1}{x^{\sigma}}\left(\theta_{x} \in\left(0, \frac{1}{x}\right)\right)
$$

This proves Inequality (5).

Lemma 2. The following reverse inequality is valid:

$$
\begin{aligned}
I= & \int_{0}^{\infty} \sum_{n=1}^{\infty} \frac{f(x) a_{n}}{(x+n)^{\lambda}} d x>B^{\frac{1}{p}}(\sigma, \lambda-\sigma) B^{\frac{1}{q}}(\mu, \lambda-\mu) \\
& \times\left\{\int_{0}^{\infty}\left(1-\rho_{\sigma}(x)\right) x^{p\left[1-\left(\frac{\lambda-\sigma}{p}+\frac{\mu}{q}\right)\right]-1} f^{p}(x) d x\right\}^{\frac{1}{p}}\left\{\sum_{n=1}^{\infty} n^{q\left[1-\left(\frac{\sigma}{p}+\frac{\lambda-\mu}{q}\right)\right]-1} a_{n}^{q}\right\}^{\frac{1}{q}} .
\end{aligned}
$$

Proof. For $n \in \mathbf{N}$, setting $x=n u$, we obtain the following weight function:

$$
\omega(\mu, n):=n^{\lambda-\mu} \int_{0}^{\infty} \frac{x^{\mu-1} d x}{(x+n)^{\lambda}}=\int_{0}^{\infty} \frac{u^{\mu-1} d u}{(u+1)^{\lambda}}=B(\mu, \lambda-\mu) .
$$


For $0<p<1, q<0$, by the reverse Hölder's inequality (cf., [27]) and the Lebesgue term by term integration theorem (cf., [28]), we obtain

$$
\begin{aligned}
& \int_{0}^{\infty} \sum_{n=1}^{\infty} \frac{f(x) a_{n}}{(x+n)^{\lambda}} d x=\int_{0}^{\infty} \sum_{n=1}^{\infty} \frac{1}{(x+n)^{\lambda}}\left[\frac{n^{(\sigma-1) / p}}{x^{(\mu-1) / q}} f(x)\right]\left[\frac{x^{(\mu-1) / q}}{n^{(\sigma-1) / p}} a_{n}\right] d x \\
\geq & \left\{\int_{0}^{\infty}\left[\sum_{n=1}^{\infty} \frac{1}{(x+n)^{\lambda}} \frac{n^{\sigma-1}}{x^{(\mu-1)(p-1)}}\right] f^{p}(x) d x\right\}^{\frac{1}{p}}\left\{\sum_{n=1}^{\infty}\left[\int_{0}^{\infty} \frac{1}{(x+n)^{\lambda}} \frac{x^{\mu-1}}{n^{(\sigma-1)(q-1)}} d x\right] a_{n}^{q}\right\}^{\frac{1}{q}} \\
= & \left\{\int_{0}^{\infty} \omega(\sigma, x) x^{p\left[1-\left(\frac{\lambda-\sigma}{p}+\frac{\mu}{q}\right)\right]-1} f^{p}(x) d x\right\}^{\frac{1}{p}}\left\{\sum_{n=1}^{\infty} \omega(\mu, n) n^{q\left[1-\left(\frac{\sigma}{p}+\frac{\lambda-\mu}{q}\right)\right]-1} a_{n}^{q}\right\}^{\frac{1}{q}} .
\end{aligned}
$$

Then by (5) and (7), we obtain Inequality (6).

Remark 1. For $\mu+\sigma=\lambda$, we find

$$
\begin{gathered}
\omega(\sigma, x)=x^{\mu} \sum_{n=1}^{\infty} \frac{n^{\sigma-1}}{(x+n)^{\lambda}}\left(x \in \mathrm{R}_{+}\right), \\
0<\int_{0}^{\infty} x^{p(1-\mu)-1} f^{p}(x) d x<\infty \text { and } 0<\sum_{n=1}^{\infty} n^{q(1-\sigma)-1} a_{n}^{q}<\infty,
\end{gathered}
$$

and then we reduce (6) as follows:

$$
\int_{0}^{\infty} \sum_{n=1}^{\infty} \frac{f(x) a_{n}}{(x+n)^{\lambda}} d x>B(\mu, \sigma)\left[\int_{0}^{\infty}\left(1-\rho_{\sigma}(x)\right) x^{p(1-\mu)-1} f^{p}(x) d x\right]^{\frac{1}{p}}\left[\sum_{n=1}^{\infty} n^{q(1-\sigma)-1} a_{n}^{q}\right]^{\frac{1}{q}} .
$$

Lemma 3. The constant factor $B(\mu, \sigma)$ in (8) is the best possible.

Proof. For $0<\varepsilon<p \mu$, we set

$$
\widetilde{f}(x):=\left\{\begin{array}{c}
0,0<x<1, \\
x^{\mu-\frac{\varepsilon}{p}-1}, x \geq 1
\end{array}, \widetilde{a}_{n}:=n^{\sigma-\frac{\varepsilon}{q}-1}(n \in \mathrm{N}) .\right.
$$

If there exists a positive constant $M(M \geq B(\mu, \sigma))$ such that (8) is valid when replacing $B(\mu, \sigma)$ by $M$, then by a substitution of $f(x)=\widetilde{f}(x), a_{n}=\widetilde{a}_{n}$, we get

$$
\begin{gathered}
\widetilde{I}:=\int_{0}^{\infty} \sum_{n=1}^{\infty} \frac{\widetilde{f}(x) \widetilde{a}_{n}}{(x+n)^{\lambda}} d x>M \\
\times\left[\int_{0}^{\infty}\left(1-\rho_{\sigma}(x)\right) x^{p(1-\mu)-1} \widetilde{f^{p}}(x) d x\right]^{\frac{1}{p}}\left[\sum_{n=1}^{\infty} n^{q(1-\sigma)-1} \widetilde{a}_{n}^{q}\right]^{\frac{1}{q}} \\
=M\left(\int_{1}^{\infty}\left(1-O\left(\frac{1}{x^{\sigma}}\right)\right) x^{-\varepsilon-1} d x\right)^{\frac{1}{p}}\left(\sum_{n=1}^{\infty} n^{-\varepsilon-1}\right)^{\frac{1}{q}} \\
\geq M\left(\int_{1}^{\infty} x^{-\varepsilon-1} d x-\int_{1}^{\infty} O\left(\frac{1}{x^{\sigma+\varepsilon+1}}\right) d x\right)^{\frac{1}{p}}\left(\int_{1}^{\infty} x^{-\varepsilon-1} d x\right)^{\frac{1}{q}} \\
=\frac{M}{\varepsilon}(1-\varepsilon O(1))^{\frac{1}{p}} .
\end{gathered}
$$


For $\mu-\frac{\varepsilon}{p}>0(0<p<1)$, by (7), we obtain

$$
\begin{aligned}
\widetilde{I} & =\sum_{n=1}^{\infty} n^{-\varepsilon-1}\left[n^{\left(\sigma+\frac{\varepsilon}{p}\right)} \int_{1}^{\infty} \frac{x^{\left(\mu-\frac{\varepsilon}{p}\right)-1}}{(x+n)^{\lambda}} d x\right] \leq \sum_{n=1}^{\infty} n^{-\varepsilon-1}\left[n^{\left(\sigma+\frac{\varepsilon}{p}\right)} \int_{0}^{\infty} \frac{x^{\left(\mu-\frac{\varepsilon}{p}\right)-1}}{(x+n)^{\lambda}} d x\right] \\
& =\sum_{n=1}^{\infty} n^{-\varepsilon-1} \omega\left(\mu-\frac{\varepsilon}{p}, n\right)=B\left(\mu-\frac{\varepsilon}{p}, \sigma+\frac{\varepsilon}{p}\right)\left(1+\sum_{n=2}^{\infty} n^{-\varepsilon-1}\right) \\
& \leq B\left(\mu-\frac{\varepsilon}{p}, \sigma+\frac{\varepsilon}{p}\right)\left(1+\int_{1}^{\infty} x^{-\varepsilon-1} d x\right)=\frac{\varepsilon+1}{\varepsilon} B\left(\mu-\frac{\varepsilon}{p}, \sigma+\frac{\varepsilon}{p}\right)
\end{aligned}
$$

Then we have

$$
(\varepsilon+1) B\left(\mu-\frac{\varepsilon}{p}, \sigma+\frac{\varepsilon}{p}\right) \geq \varepsilon \widetilde{I} \geq M(1-\varepsilon O(1))^{\frac{1}{p}} .
$$

For $\varepsilon \rightarrow 0^{+}$, in view of the continuity of the beta function, it follows that $B(\mu, \sigma) \geq M$. Therefore, $M=B(\mu, \sigma)$ is the best possible constant factor of (8). Lemma 3 is proved.

Remark 2. Setting $\hat{\mu}:=\frac{\lambda-\sigma}{p}+\frac{\mu}{q}, \hat{\sigma}:=\frac{\sigma}{p}+\frac{\lambda-\mu}{q}$, we have

$$
\hat{\mu}+\hat{\sigma}=\frac{\lambda-\sigma}{p}+\frac{\mu}{q}+\frac{\sigma}{p}+\frac{\lambda-\mu}{q}=\frac{\lambda}{p}+\frac{\lambda}{q}=\lambda,
$$

and for $\lambda-\mu-\sigma \in(-p \mu, p(\lambda-\mu))$, we find

$$
\begin{aligned}
& \hat{\mu}>\frac{(1-p) \mu}{p}+\frac{\mu}{q}=0, \hat{\mu}<\frac{\mu+p(\lambda-\mu)}{p}+\frac{\mu}{q}=\lambda, \\
& 0<\hat{\sigma}=\lambda-\hat{\mu}<\lambda, B(\hat{\mu}, \hat{\sigma}) \in \mathrm{R}_{+} .
\end{aligned}
$$

We can reduce (6) to the following

$$
\begin{aligned}
\int_{0}^{\infty} & \sum_{n=1}^{\infty} \frac{f(x) a_{n}}{(x+n)^{\lambda}} d x>B^{\frac{1}{p}}(\sigma, \lambda-\sigma) B^{\frac{1}{q}}(\mu, \lambda-\mu) \\
& \times\left[\int_{0}^{\infty}\left(1-\rho_{\sigma}(x)\right) x^{p(1-\hat{\mu})-1} f^{p}(x) d x\right]^{\frac{1}{p}}\left[\sum_{n=1}^{\infty} n^{q(1-\hat{\sigma})-1} a_{n}^{q}\right]^{\frac{1}{q}} .
\end{aligned}
$$

Lemma 4. If $\lambda-\mu-\sigma \in(-p \mu, p(\lambda-\mu))$, the constant factor $B^{\frac{1}{p}}(\sigma, \lambda-\sigma) B^{\frac{1}{q}}(\mu, \lambda-\mu)$ in (9) is the best possible, then we have $\mu+\sigma=\lambda$.

Proof. If the constant factor $B^{\frac{1}{p}}(\sigma, \lambda-\sigma) B^{\frac{1}{q}}(\mu, \lambda-\mu)$ in (9) is the best possible, then by (8), the unique best possible constant factor must be $B(\hat{\mu}, \hat{\sigma})\left(\in \mathrm{R}_{+}\right)$, namely,

$$
B(\hat{\mu}, \hat{\sigma})=B^{\frac{1}{p}}(\sigma, \lambda-\sigma) B^{\frac{1}{q}}(\mu, \lambda-\mu)
$$

By the reverse Hölder's inequality (cf., [27]), we find

$$
\begin{aligned}
B(\hat{\mu}, \hat{\sigma}) & =\int_{0}^{\infty} \frac{t^{\hat{\mu}-1}}{(1+t)^{\lambda}} d t=\int_{0}^{\infty} \frac{t^{\frac{\lambda-\sigma}{p}+\frac{\mu}{q}-1}}{(1+t)^{\lambda}} d t=\int_{0}^{\infty} \frac{1}{(1+t)^{\lambda}}\left(t^{\frac{\lambda-\sigma-1}{p}}\right)\left(t^{\frac{\mu-1}{q}}\right) d t \\
& \geq\left[\int_{0}^{\infty} \frac{1}{(1+t)^{\lambda}} t^{\lambda-\sigma-1} d t\right]^{\frac{1}{p}}\left[\int_{0}^{\infty} \frac{1}{(1+t)^{\lambda}} t^{\mu-1} d t\right]^{\frac{1}{q}} \\
& =B^{\frac{1}{p}}(\sigma, \lambda-\sigma) B^{\frac{1}{q}}(\mu, \lambda-\mu) .
\end{aligned}
$$

We observe that (10) keeps the form of equality if and only if there exist constants $A, B$ such that they are not all zero and

$$
A t^{\lambda-\sigma-1}=B t^{\mu-1} \text { a.e. in } \mathrm{R}_{+} \text {. }
$$


Suppose that $A \neq 0$. We find that $t^{\lambda-\mu-\sigma}=\frac{B}{A}$ a.e. in $\mathbf{R}_{+}$, and thus we conclude that $\lambda-\mu-\sigma=0$, i.e., $\mu+\sigma=\lambda$. Lemma 4 is proved.

\section{Main Results}

Theorem 1. Inequality (6) is equivalent to the following inequalities:

$$
\begin{gathered}
J_{1}:=\left\{\sum_{n=1}^{\infty} n^{p\left(\frac{\sigma}{p}+\frac{\lambda-\mu}{q}\right)-1}\left[\int_{0}^{\infty} \frac{f(x)}{(x+n)^{\lambda}} d x\right]^{p}\right\}^{\frac{1}{p}} \\
>B^{\frac{1}{p}}(\sigma, \lambda-\sigma) B^{\frac{1}{q}}(\mu, \lambda-\mu)\left\{\int_{0}^{\infty}\left(1-\rho_{\sigma}(x)\right) x^{p\left[1-\left(\frac{\lambda-\sigma}{p}+\frac{\mu}{q}\right)\right]-1} f^{p}(x) d x\right\}^{\frac{1}{p}}, \\
J_{2}:=\left\{\int_{0}^{\infty} \frac{x^{q\left(\frac{\lambda-\sigma}{p}+\frac{\mu}{q}\right)-1}}{\left(1-\rho_{\sigma}(x)\right)^{q-1}}\left[\sum_{n=1}^{\infty} \frac{a_{n}}{(x+n)^{\lambda}}\right]^{q} d x\right\}^{\frac{1}{q}} \\
>B^{\frac{1}{p}}(\sigma, \lambda-\sigma) B^{\frac{1}{q}}(\mu, \lambda-\mu)\left\{\sum_{n=1}^{\infty} n^{q\left[1-\left(\frac{\sigma}{p}+\frac{\lambda-\mu}{q}\right)\right]-1} a_{n}^{q}\right\}^{\frac{1}{q}} .
\end{gathered}
$$

If the constant factor $B^{\frac{1}{p}}(\sigma, \lambda-\sigma) B^{\frac{1}{q}}(\mu, \lambda-\mu)$ in (6) is the best possible, then so is the constant factor in (11) and (12).

In particular, for $\mu+\sigma=\lambda$ in (6), (11) and (12), we have Inequality (8) and the following equivalent versions of reverse inequalities with the best possible constant factor $B(\mu, \sigma)$ :

$$
\begin{gathered}
\left\{\sum_{n=1}^{\infty} n^{p \sigma-1}\left[\int_{0}^{\infty} \frac{f(x)}{(x+n)^{\lambda}} d x\right]^{p \frac{1}{p}}>B(\mu, \sigma)\left[\int_{0}^{\infty}\left(1-\rho_{\sigma}(x)\right) x^{p(1-\mu)-1} f^{p}(x) d x\right]^{\frac{1}{p}},\right. \\
\left\{\int_{0}^{\infty} \frac{x^{q \mu-1}}{\left(1-\rho_{\sigma}(x)\right)^{q-1}}\left[\sum_{n=1}^{\infty} \frac{a_{n}}{(x+n)^{\lambda}}\right]^{q} d x\right\}^{\frac{1}{q}}>B(\mu, \sigma)\left[\sum_{n=1}^{\infty} n^{q(1-\sigma)-1} a_{n}^{q}\right]^{\frac{1}{q}} .
\end{gathered}
$$

Proof. Suppose that (11) is valid. By the Lebesgue term by term integration theorem and the reverse Hölder's inequality (cf., [27,28]), we have

$$
\begin{aligned}
I & =\sum_{n=1}^{\infty} \int_{0}^{\infty} \frac{f(x) a_{n}}{(x+n)^{\lambda}} d x=\sum_{n=1}^{\infty}\left[n^{\frac{-1}{p}+\left(\frac{\sigma}{p}+\frac{\lambda-\mu}{q}\right)} \int_{0}^{\infty} \frac{f(x)}{(x+n)^{\lambda}} d x\right]\left[n^{\frac{1}{p}-\left(\frac{\sigma}{p}+\frac{\lambda-\mu}{q}\right)} a_{n}\right] \\
& \geq J_{1}\left\{\sum_{n=1}^{\infty} n^{q\left[1-\left(\frac{\sigma}{p}+\frac{\lambda-\mu}{q}\right)\right]-1} a_{n}^{q}\right\}^{\frac{1}{q}} .
\end{aligned}
$$

Then by (11), we have Inequality (6). On the other-hand, assuming that Inequality (6) is valid, we set

$$
a_{n}:=n^{p\left(\frac{\sigma}{p}+\frac{\lambda-\mu}{q}\right)-1}\left[\int_{0}^{\infty} \frac{f(x)}{(x+n)^{\lambda}} d x\right]^{p-1}, n \in \mathrm{N} .
$$

If $J_{1}=\infty$, then Inequality (11) is naturally valid; if $J_{1}=0$, so it is impossible to make Inequality (11) valid, namely $J_{1}>0$. Suppose that $0<J_{1}<\infty$. By (6), we have

$$
\begin{aligned}
& \sum_{n=1}^{\infty} n^{q\left[1-\left(\frac{\sigma}{p}+\frac{\lambda-\mu}{q}\right)\right]-1} a_{n}^{q}=J_{1}^{p}=I>B^{\frac{1}{p}}(\sigma, \lambda-\sigma) B^{\frac{1}{q}}(\mu, \lambda-\mu) \\
& \quad \times\left\{\int_{0}^{\infty}\left(1-\rho_{\sigma}(x)\right) x^{p\left[1-\left(\frac{\lambda-\sigma}{p}+\frac{\mu}{q}\right)\right]-1} f^{p}(x) d x\right\}^{\frac{1}{p}}\left\{\sum_{n=1}^{\infty} n^{q\left[1-\left(\frac{\sigma}{p}+\frac{\lambda-\mu}{q}\right)\right]-1} a_{n}^{q}\right\}^{\frac{1}{q}},
\end{aligned}
$$




$$
\begin{aligned}
& \left\{\sum_{n=1}^{\infty} n^{q\left[1-\left(\frac{\sigma}{p}+\frac{\lambda-\mu}{q}\right)\right]-1} a_{n}^{q}\right\}^{\frac{1}{p}}=J_{1}>B^{\frac{1}{p}}(\sigma, \lambda-\sigma) B^{\frac{1}{q}}(\mu, \lambda-\mu) \\
& \quad \times\left\{\int_{0}^{\infty}\left(1-\rho_{\sigma}(x)\right) x^{p\left[1-\left(\frac{\lambda-\sigma}{p}+\frac{\mu}{q}\right)\right]-1} f^{p}(x) d x\right\}^{\frac{1}{p}}
\end{aligned}
$$

namely, Inequality (11) follows, which is equivalent to Inequality (6).

Suppose that Inequality (12) is valid. By the reverse Hölder's inequality, we have

$$
\begin{aligned}
I & =\int_{0}^{\infty}\left[\left(1-\rho_{\sigma}(x)\right)^{\frac{1}{p}} x^{\frac{1}{q}-\left(\frac{\lambda-\sigma}{p}+\frac{\mu}{q}\right)} f(x)\right]\left[\frac{x^{\frac{-1}{q}+\left(\frac{\lambda-\sigma}{p}+\frac{\mu}{q}\right)}}{\left(1-\rho_{\sigma}(x)\right)^{1 / p}} \sum_{n=1}^{\infty} \frac{a_{n}}{(x+n)^{\lambda}}\right] d x \\
& \geq\left\{\int_{0}^{\infty}\left(1-\rho_{\sigma}(x)\right) x^{p\left\{1-\left(\frac{\lambda-\sigma}{p}+\frac{\mu}{q}\right)\right]-1} f^{p}(x) d x\right\}^{\frac{1}{p}} J_{2} .
\end{aligned}
$$

Then by (12), we obtain Inequality (6). On the other-hand, assuming that Inequality (6) is valid, we set

$$
f(x):=x^{q\left(\frac{\lambda-\sigma}{p}+\frac{\mu}{q}\right)-1}\left[\sum_{n=1}^{\infty} \frac{a_{n}}{(x+n)^{\lambda}}\right]^{q-1}, x \in \mathrm{R}_{+}
$$

If $J_{2}=\infty$, then Inequality (12) is naturally valid; if $J_{2}=0$, then it is impossible to make Inequality (12) valid, namely $J_{2}>0$. Suppose that $0<J_{2}<\infty$. By (6), we have

$$
\begin{gathered}
\int_{0}^{\infty}\left(1-\rho_{\sigma}(x)\right) x^{p\left[1-\left(\frac{\lambda-\sigma}{p}+\frac{\mu}{q}\right)\right]-1} f^{p}(x) d x=J_{2}^{q}=I>B^{\frac{1}{p}}(\sigma, \lambda-\sigma) B^{\frac{1}{q}}(\mu, \lambda-\mu) \\
\times\left\{\int_{0}^{\infty}\left(1-\rho_{\sigma}(x)\right) x^{p\left[1-\left(\frac{\lambda-\sigma}{p}+\frac{\mu}{q}\right)\right]-1} f^{p}(x) d x\right\}^{\frac{1}{p}}\left\{\sum_{n=1}^{\infty} n^{q\left[1-\left(\frac{\sigma}{p}+\frac{\lambda-\mu}{q}\right)\right]-1} a_{n}^{q}\right\}^{\frac{1}{q}}, \\
\left\{\int_{0}^{\infty}\left(1-\rho_{\sigma}(x)\right) x^{p\left[1-\left(\frac{\lambda-\sigma}{p}+\frac{\mu}{q}\right)\right]-1} f^{p}(x) d x\right\}^{\frac{1}{q}}=J_{2}>B^{\frac{1}{p}}(\sigma, \lambda-\sigma) B^{\frac{1}{q}}(\mu, \lambda-\mu) \\
\times\left\{\sum_{n=1}^{\infty} n^{q\left[1-\left(\frac{\sigma}{p}+\frac{\lambda-\mu}{q}\right)\right]-1} a_{n}^{q}\right\}^{\frac{1}{q}},
\end{gathered}
$$

namely, Inequality (12) follows, which is equivalent to Inequality (6).

Hence, Inequalities (6), (11) and (12) are equivalent.

If the constant factor $B^{\frac{1}{p}}(\sigma, \lambda-\sigma) B^{\frac{1}{q}}(\mu, \lambda-\mu)$ in (6) is the best possible, then so is the constant factor in (11) and (12). Otherwise, by (15) (or (16)), we would reach a contradiction that the constant factor in (6) is not the best possible. This completes the proof of Theorem 1.

Theorem 2. The following statements (i), (ii), (iii) and (iv) are equivalent.

(i) $B^{\frac{1}{p}}(\sigma, \lambda-\sigma) B^{\frac{1}{q}}(\mu, \lambda-\mu)$ is independent of $p, q$;

(ii) $B^{\frac{1}{p}}(\sigma, \lambda-\sigma) B^{\frac{1}{q}}(\mu, \lambda-\mu)$ is expressible as a single integral;

(iii) $B^{\frac{1}{p}}(\sigma, \lambda-\sigma) B^{\frac{1}{q}}(\mu, \lambda-\mu)$ is the best possible of (6);

(iv) If $\lambda-\mu-\sigma \in(-p \mu, p(\lambda-\mu))$, then $\mu+\sigma=\lambda$.

Proof. (i) $\Rightarrow$ (ii). In view of $B^{\frac{1}{p}}(\sigma, \lambda-\sigma) B^{\frac{1}{q}}(\mu, \lambda-\mu)$ is independent of $p, q$, we find

$$
\begin{aligned}
& B^{\frac{1}{p}}(\sigma, \lambda-\sigma) B^{\frac{1}{q}}(\mu, \lambda-\mu) \\
= & \lim _{\substack{p \rightarrow 1^{-},\\
}} B^{\frac{1}{p}}(\sigma, \lambda-\sigma) B^{\frac{1}{q}}(\mu, \lambda-\mu)=B(\sigma, \lambda-\sigma), \\
&
\end{aligned}
$$

which is a single integral $\int_{0}^{\infty} \frac{t^{\sigma-1}}{(1+t)^{\lambda}} d t$ 
(ii) $\Rightarrow$ (iv). Suppose that $B^{\frac{1}{p}}(\sigma, \lambda-\sigma) B^{\frac{1}{q}}(\mu, \lambda-\mu)$ is expressible as a single integral $\int_{0}^{\infty} \frac{t^{\frac{\lambda-\sigma}{p}+\frac{\mu}{q}-1}}{(1+t)^{\lambda}} d t$. Then (10) keeps the form of equality. By the proof of Lemma 4 , for $\lambda-\mu-\sigma \in(-p \mu, p(\lambda-\mu))$, we have $\mu+\sigma=\lambda$.

(iv) $\Rightarrow$ (i). If $\mu+\sigma=\lambda$, then

$$
B^{\frac{1}{p}}(\sigma, \lambda-\sigma) B^{\frac{1}{q}}(\mu, \lambda-\mu)=B(\mu, \sigma),
$$

which is independent of $p, q$.

Hence, (i) $\Leftrightarrow$ (ii) $\Leftrightarrow$ (iv).

(vi) $\Rightarrow$ (iii). By Lemma 3, for $\mu+\sigma=\lambda, B^{\frac{1}{p}}(\sigma, \lambda-\sigma) B^{\frac{1}{q}}(\mu, \lambda-\mu)$ is the best possible of (6).

(iii) $\Rightarrow$ (iv). By Lemma 4, we have $\mu+\sigma=\lambda$.

Therefore, we show that (iv ) $\Leftrightarrow$ (iii), and then the statements (i), (ii), (iii) and (iv) are equivalent. The proof Theorem 2 is complete.

\section{Two Corollaries and Some Particular Inequalities}

Replacing $x$ by $\frac{1}{x}$, and then setting $F(x)=x^{\lambda-2} f\left(\frac{1}{x}\right)$ in Theorems 1 and 2, we find

$$
\rho_{\sigma}\left(x^{-1}\right)=\frac{\left(1+\theta_{x^{-1}}\right)^{-\lambda}}{\sigma B(\sigma, \lambda-\sigma)} x^{\sigma}=O\left(x^{\sigma}\right) \in(0,1)\left(\theta_{x^{-1}} \in(0, x) ; x>0\right),
$$

and obtain the following corollaries:

Corollary 1. If $F(x), a_{n} \geq 0$ such that

$$
0<\int_{0}^{\infty} x^{p\left[1-\left(\frac{\sigma}{p}+\frac{\lambda-\mu}{q}\right)\right]-1} F^{p}(x) d x<\infty \text { and } 0<\sum_{n=1}^{\infty} n^{q\left[1-\left(\frac{\sigma}{p}+\frac{\lambda-\mu}{q}\right)\right]-1} a_{n}^{q}<\infty,
$$

then the following inequalities are equivalent:

$$
\begin{gathered}
\int_{0}^{\infty} \sum_{n=1}^{\infty} \frac{F(x) a_{n}}{(1+x n)^{\lambda}} d x>B^{\frac{1}{p}}(\sigma, \lambda-\sigma) B^{\frac{1}{q}}(\mu, \lambda-\mu) \\
\times\left\{\int_{0}^{\infty}\left(1-\rho_{\sigma}\left(x^{-1}\right)\right) x^{p\left[1-\left(\frac{\sigma}{p}+\frac{\lambda-\mu}{q}\right)\right]-1} F^{p}(x) d x\right\}^{\frac{1}{p}}\left\{\sum_{n=1}^{\infty} n^{q\left[1-\left(\frac{\sigma}{p}+\frac{\lambda-\mu}{q}\right)\right]-1} a_{n}^{q}\right\}^{\frac{1}{q}}, \\
\left\{\sum_{n=1}^{\infty} n^{p\left(\frac{\sigma}{p}+\frac{\lambda-\mu}{q}\right)-1}\left[\int_{0}^{\infty} \frac{F(x)}{(1+x n)^{\lambda}} d x\right]^{p}\right\}^{\frac{1}{p}} \\
>B^{\frac{1}{p}}(\sigma, \lambda-\sigma) B^{\frac{1}{q}}(\mu, \lambda-\mu)\left\{\int_{0}^{\infty}\left(1-\rho_{\sigma}\left(x^{-1}\right)\right) x^{p\left[1-\left(\frac{\sigma}{p}+\frac{\lambda-\mu}{q}\right)\right]-1} F^{p}(x) d x\right\}^{\frac{1}{p}}, \\
\left\{\int_{0}^{\infty} \frac{x^{q\left(\frac{\sigma}{p}+\frac{\lambda-\mu}{q}\right)-1}}{\left(1-\rho_{\sigma}\left(x^{-1}\right)\right)^{q-1}}\left[\sum_{n=1}^{\infty} \frac{a_{n}}{(1+x n)^{\lambda}}\right] d x\right\}^{\frac{1}{q}} \\
>B^{\frac{1}{p}}(\sigma, \lambda-\sigma) B^{\frac{1}{q}}(\mu, \lambda-\mu)\left\{\sum_{n=1}^{\infty} n^{q\left[1-\left(\frac{\sigma}{p}+\frac{\lambda-\mu}{q}\right)\right]-1} a_{n}^{q}\right\}^{\frac{1}{q}} .
\end{gathered}
$$

If the constant factor $B^{\frac{1}{p}}(\sigma, \lambda-\sigma) B^{\frac{1}{q}}(\mu, \lambda-\mu)$ in (17) is the best possible, then so is the constant factor in (18) and (19). 
In particular, for $\mu=\lambda-\sigma$ in (17), (18) and (19), we have the following equivalent inequalities with the best possible constant factor $B(\lambda-\sigma, \sigma)$ :

$$
\begin{aligned}
& \int_{0}^{\infty} \sum_{n=1}^{\infty} \frac{F(x) a_{n}}{(1+x n)^{\lambda}} d x>B(\lambda-\sigma, \sigma) \\
& \times {\left[\int_{0}^{\infty}\left(1-\rho_{\sigma}\left(x^{-1}\right)\right) x^{p(1-\sigma)-1} F^{p}(x) d x\right]^{\frac{1}{p}}\left[\sum_{n=1}^{\infty} n^{q(1-\sigma)-1} a_{n}^{q}\right]^{\frac{1}{q}}, } \\
&\left\{\sum_{n=1}^{\infty} n^{p \sigma-1}\left[\int_{0}^{\infty} \frac{F(x)}{(1+x n)^{\lambda}} d x\right]^{p}\right\}^{\frac{1}{p}} \\
&>B(\lambda-\sigma, \sigma)\left[\int_{0}^{\infty}\left(1-\rho_{\sigma}\left(x^{-1}\right)\right) x^{p(1-\sigma)-1} F^{p}(x) d x\right]^{\frac{1}{p}}, \\
&\left\{\int_{0}^{\infty} \frac{x^{q \sigma-1}}{\left(1-\rho\left(x^{-1}\right)\right)^{q-1}}\left[\sum_{n=1}^{\infty} \frac{a_{n}}{(1+x n)^{\lambda}}\right]^{q} d x\right\}^{\frac{1}{q}}>B(\lambda-\sigma, \sigma)\left[\sum_{n=1}^{\infty} n^{q(1-\sigma)-1} a_{n}^{q}\right]^{\frac{1}{q}} .
\end{aligned}
$$

Corollary 2. The following statements (i), (ii), (iii) and (iv) are equivalent:

(i) $B^{\frac{1}{p}}(\sigma, \lambda-\sigma) B^{\frac{1}{q}}(\mu, \lambda-\mu)$ is independent of $p, q$;

(ii) $B^{\frac{1}{p}}(\sigma, \lambda-\sigma) B^{\frac{1}{q}}(\mu, \lambda-\mu)$ is expressible as a single integral;

(iii) $B^{\frac{1}{p}}(\sigma, \lambda-\sigma) B^{\frac{1}{q}}(\mu, \lambda-\mu)$ is the best possible of (17);

(iv) If $\lambda-\mu-\sigma \in(-q \sigma, q(\lambda-\sigma))$, then we have $\mu=\lambda-\sigma$.

Remark 3. (i) For $\sigma=2<\lambda(\leq 5), \mu=\lambda-2$ in (8), (13) and (14), since

$$
\begin{gathered}
B(\lambda-2,2)=\frac{\Gamma(\lambda-2) \Gamma(2)}{\Gamma(\lambda)}=\frac{\Gamma(\lambda-2)}{(\lambda-1)(\lambda-2) \Gamma(\lambda-2)}=\frac{1}{(\lambda-1)(\lambda-2)}, \\
\rho_{2}(x)=\frac{(\lambda-1)(\lambda-2)}{2\left(1+\theta_{x}\right)^{\lambda}} \frac{1}{x^{2}}=O\left(\frac{1}{x^{2}}\right) \in(0,1)\left(\theta_{x} \in\left(0, \frac{1}{x}\right) ; x>0\right),
\end{gathered}
$$

we have the following equivalent versions of reverse inequalities with the best possible constant factor $\frac{1}{(\lambda-1)(\lambda-2)}$ :

$$
\begin{gathered}
\int_{0}^{\infty} \sum_{n=1}^{\infty} \frac{f(x) a_{n}}{(x+n)^{\lambda}} d x>\frac{1}{(\lambda-1)(\lambda-2)}\left[\int_{0}^{\infty}\left(1-\rho_{2}(x)\right) x^{p(3-\lambda)-1} f^{p}(x) d x\right]^{\frac{1}{p}}\left(\sum_{n=1}^{\infty} n^{-q-1} a_{n}^{q}\right)^{\frac{1}{q}}, \\
\left\{\sum_{n=1}^{\infty} n^{2 p-1}\left[\int_{0}^{\infty} \frac{f(x)}{(x+n)^{\lambda}} d x\right]\right\}^{\frac{1}{p}}>\frac{1}{(\lambda-1)(\lambda-2)}\left[\int_{0}^{\infty}\left(1-\rho_{2}(x)\right) x^{p(3-\lambda)-1} f^{p}(x) d x\right]^{\frac{1}{p}}, \\
\left\{\int_{0}^{\infty} \frac{x^{q(\lambda-2)-1}}{\left(1-\rho_{2}(x)\right)^{q-1}}\left[\sum_{n=1}^{\infty} \frac{a_{n}}{(x+n)^{\lambda}}\right]^{q} d x\right\}^{\frac{1}{q}}>\frac{1}{(\lambda-1)(\lambda-2)}\left(\sum_{n=1}^{\infty} n^{-q-1} a_{n}^{q}\right)^{\frac{1}{q}} .
\end{gathered}
$$

(ii) For $\sigma=2<\lambda(\leq 5), \mu=\lambda-2$ in (20), (21) and (22), we have

$$
\rho_{2}\left(x^{-1}\right)=\frac{(\lambda-1)(\lambda-2)}{2\left(1+\theta_{x^{-1}}\right)^{\lambda}} x^{2}=O\left(x^{2}\right) \in(0,1)\left(\theta_{x^{-1}} \in(0, x) ; x>0\right),
$$

and the following equivalent versions of reverse inequalities with the best possible constant factor $\frac{1}{(\lambda-1)(\lambda-2)}$ :

$$
\int_{0}^{\infty} \sum_{n=1}^{\infty} \frac{F(x) a_{n}}{(1+x n)^{\lambda}} d x>\frac{1}{(\lambda-1)(\lambda-2)}\left(\int_{0}^{\infty}\left(1-\rho_{2}\left(x^{-1}\right)\right) x^{-p-1} F^{p}(x) d x\right)^{\frac{1}{p}}\left(\sum_{n=1}^{\infty} n^{-q-1} a_{n}^{q}\right)^{\frac{1}{q}},
$$




$$
\begin{gathered}
\left\{\sum_{n=1}^{\infty} n^{2 p-1}\left[\int_{0}^{\infty} \frac{F(x)}{(1+x n)^{\lambda}} d x\right]^{p\}^{\frac{1}{p}}>} \frac{1}{(\lambda-1)(\lambda-2)}\left(\int_{0}^{\infty}\left(1-\rho_{2}\left(x^{-1}\right)\right) x^{-p-1} F^{p}(x) d x\right)^{\frac{1}{p}},\right. \\
\left\{\int_{0}^{\infty} \frac{x^{2 q-1}}{\left(1-\rho_{2}\left(x^{-1}\right)\right)^{q-1}}\left[\sum_{n=1}^{\infty} \frac{a_{n}}{(1+x n)^{\lambda}}\right] d x\right\}^{\frac{1}{q}}
\end{gathered}
$$

\section{Conclusions}

Let us give a brief summary of this paper, by the way of $[20,22]$ and the use of the weight functions, the idea of introducing parameters and the Euler-Maclaurin summation formula, a reverse half-discrete Hardy-Hilbert's inequality and the reverse equivalent forms are given in Lemma 2 and Theorem 1. The equivalent statements of the best possible constant factor related to some parameters are proved in Theorem 2. As applications, two corollaries about the reverse cases of the non-homogeneous kernel and some particular cases are considered in Corollaries 1,2 and Remark 3. The above-mentioned lemmas and theorems reveal some essential characters of this type of Hardy-Hilbert inequality.

Author Contributions: B.Y. carried out the mathematical studies, participated in the sequence alignment and drafted the manuscript. S.W. and A.W. participated in the design of the study and performed the numerical analysis. All authors read and approved the final manuscript.

Funding: This work is supported by the National Natural Science Foundation (No. 61772140), and Science and Technology Planning Project Item of Guangzhou City (No. 201707010229). We are grateful for their help.

Acknowledgments: The authors thanks the referees for their useful proposes for reforming the paper.

Conflicts of Interest: The authors declare that they have no competing interest.

\section{References}

1. Hardy, G.H.; Littlewood, J.E.; Polya, G. Inequalities; Cambridge University Press: Cambridge, UK, 1934.

2. Yang, B.C. The Norm of Operator and Hilbert-Type Inequalities; Science Press: Beijing, China, 2009.

3. Yang, B.C. Hilbert-Type Integral Inequalities; Bentham Science Publishers Ltd.: Sharjah, UAE, 2009.

4. Yang, B.C. On the norm of an integral operator and applications. J. Math. Anal. Appl. 2006, 321, $182-192$. [CrossRef]

5. Xu, J.S. Hardy-Hilbert's inequalities with two parameters. Adv. Math. 2007, 36, 63-76.

6. Yang, B.C. On the norm of a Hilbert's type linear operator and applications. J. Math. Anal. Appl. 2007, 325, 529-541. [CrossRef]

7. Xie, Z.T.; Zeng, Z.; Sun, Y.F. A new Hilbert-type inequality with the homogeneous kernel of degree -2 . Adv. Appl. Math. Sci. 2013, 12, 391-401.

8. Zhen, Z.; Raja Rama Gandhi, K.; Xie, Z.T. A new Hilbert-type inequality with the homogeneous kernel of degree -2 and with the integral. Bull. Math. Sci. Appl. 2014, 3, 11-20.

9. Xin, D.M. A Hilbert-type integral inequality with the homogeneous kernel of zero degree. Math. Theory Appl. 2010, 30, 70-74.

10. Azar, L.E. The connection between Hilbert and Hardy inequalities. J. Inequal. Appl. 2013, 452, 2013. [CrossRef]

11. Batbold, T.; Sawano, Y. Sharp bounds for m-linear Hilbert-type operators on the weighted Morrey spaces. Math. Inequal. Appl. 2017, 20, 263-283. [CrossRef]

12. Adiyasuren, V.; Batbold, T.; Krnic, M. Multiple Hilbert-type inequalities involving some differential operators. Banach. J. Math. Anal. 2016, 10, 320-337. [CrossRef]

13. Adiyasuren, V.; Batbold, T.; Krnic, M. Hilbert-type inequalities involving differential operators, the best constants and applications. Math. Inequal. Appl. 2015, 18, 111-124. [CrossRef]

14. Rassias, M.T.H.; Yang, B.C. On half-discrete Hilbert's inequality. Appl. Math. Comput. 2013, 220, 75-93. [CrossRef]

15. Yang, B.C.; Krnic, M. A half-discrete Hilbert-type inequality with a general homogeneous kernel of degree 0 . J. Math. Inequal. 2012, 6, 401-417. 
16. Rassias, M.T.H.; Yang, B.C. A multidimensional half - discrete Hilbert-type inequality and the Riemann zeta function. Appl. Math. Comput. 2013, 225, 263-277. [CrossRef]

17. Rassias, M.T.H.; Yang, B.C. On a multidimensional half-discrete Hilbert-type inequality related to the hyperbolic cotangent function. Appl. Math. Comput. 2013, 242, 800-813. [CrossRef]

18. Huang, Z.X.; Yang, B.C. On a half-discrete Hilbert-Type inequality similar to Mulholland's inequality. J. Inequal. Appl. 2013, 290, 2013. [CrossRef]

19. Yang, B.C.; Lebnath, L. Half-Discrete Hilbert-Type Inequalities; World Scientific Publishing: Singapore, 2014.

20. Krnic, M.; Pecaric, J. Extension of Hilbert's inequality. J. Math. Anal. Appl. 2006, 324, 150-160. [CrossRef]

21. Adiyasuren, V.; Batbold, T.; Azar, L.E. A new discrete Hilbert-type inequality involving partial sums. J. Inequal. Appl. 2019, 127, 2019. [CrossRef]

22. Hong, Y.; Wen, Y. A necessary and Sufficient condition of that Hilbert type series inequality with homogeneous kernel has the best constant factor. Ann. Math. 2016, 37, 329-336.

23. Hong, Y. On the structure character of Hilbert's type integral inequality with homogeneous kernel and applications. J. Jilin Univ. Sci. Ed. 2017, 55, 189-194.

24. Hong, Y.; Huang, Q.L.; Yang, B.C.; Liao, J.L. The necessary and sufficient conditions for the existence of a kind of Hilbert-type multiple integral inequality with the non-homogeneous kernel and its applications. J. Inequal. Appl. 2017, 2017, 316. [CrossRef]

25. Xin, D.M.; Yang, B.C.; Wang, A.Z. Equivalent property of a Hilbert-type integral inequality related to the beta function in the whole plane. J. Funct. Spaces 2018. [CrossRef]

26. Hong, Y.; He, B.; Yang, B.C. Necessary and Sufficient Conditions for the Validity of Hilbert Type Integral Inequalities with a Class of Quasi-Homogeneous Kernels and Its Application in Operator Theory. J. Math. Inequal. 2018, 12, 777-788. [CrossRef]

27. Kuang, J.C. Applied Inequalities; Shangdong Science and Technology Press: Jinan, China, 2004.

28. Kuang, J.C. Real and Functional Analysis (Continuation); Higher Education Press: Beijing, China, 2015.

(C) 2019 by the authors. Licensee MDPI, Basel, Switzerland. This article is an open access article distributed under the terms and conditions of the Creative Commons Attribution (CC BY) license (http://creativecommons.org/licenses/by/4.0/). 